\title{
Using Stochastic Models to Describe and Predict Social Dynamics of Web Users
}

\author{
Kristina Lerman* and Tad Hog跑
}

\begin{abstract}
Popularity of content in social media is unequally distributed, with some items receiving a disproportionate share of attention from users. Predicting which newly-submitted items will become popular is critically important for both hosts of social media content and its consumers. Accurate and timely prediction would enable hosts to maximize revenue through differential pricing for access to content or ad placement. Prediction would also give consumers an important tool for filtering the ever-growing amount of content. Predicting popularity of content in social media, however, is challenging due to the complex interactions between content quality and how the social media site chooses to highlight content. Moreover, most social media sites also selectively present content that has been highly rated by similar users, whose similarity is indicated implicitly by their behavior or explicitly by links in a social network. While these factors make it difficult to predict popularity a priori, we show that stochastic models of user behavior on these sites allows predicting popularity based on early user reactions to new content. By incorporating the various mechanisms through which web sites display content, such models improve on predictions based on simply extrapolating from the early votes. Using data from one such site, the news aggregator Digg, we show how a stochastic model of user behavior distinguishes the effect of the increased visibility due to the network from how interested users are in the content. We find a wide range of interest, identifying stories primarily of interest to users in the network ("niche interests") from those of more general interest to the user community. This distinction is useful for predicting a story's eventual popularity from users' early reactions to the story.
\end{abstract}

\section{INTRODUCTION}

Success or popularity in social media is not evenly distributed. Instead, a small number of users dominate the activity on the site and receive most of the attention of other users. The popularity of contributed items likewise shows extreme diversity. For example, relatively few of the four billion images on the social photo-sharing site Flickr are viewed thousands of times, while most of the rest are rarely viewed. Of the tens of thousands of new stories submitted daily to the social news portal Digg, only a handful go on to become wildly popular, gathering thousands of votes, while most of the remaining stories never receive more than a single vote from the submitter herself. Among thousands of new blog posts every day, only a handful become widely read and commented upon. Given the volume of new content, it is critically important to provide users with tools to help them sift through the vast stream of new content to identify interesting items in a timely manner, or least those items that will prove to be successful or popular. Accurate and timely prediction will also enable social media companies that host user-generated content to maximize revenue through differential pricing for access to content or ad placement, and encourage greater user loyalty by helping their users quickly find interesting new content.

Success in social media is difficult to predict. Although early and late popularity, which can be measured in terms of user interest, e.g., votes or views, an item generates from its inception, are somewhat correlated [13, 35], we know little about what drives success. Does success derive mainly from an item's inherent quality [2], users' response to it [10], or

\footnotetext{
*Electronic address: lerman@isi.edu

†Electronic address: tadhogg@yahoo.com
}

some external factors, such as social influence [23-25]? In a landmark study, Salganik et al. [34] addressed this question experimentally by measuring the impact of content quality and social influence on the eventual popularity or success of cultural artifacts. They showed that while quality contributes only weakly to their eventual success, social influence, or knowing about the choices of other people, is responsible for both the inequality and unpredictability of success. In their experiment, Salganik et al. asked users to rate songs they listened to. The users were assigned to different groups. In the control group (independent condition), users were simply presented with lists of songs. In the other group (social influence condition), users were also shown how many times each song was downloaded by other users. The social influence condition resulted in large inequality in popularity of songs, measured by the number of times the songs were downloaded. Although a song's quality, as measured by its popularity in the control group, was positively related to its eventual popularity in the social condition group, the variance in popularity at a given quality was very high. This means that two songs of similar quality could end up with vastly different levels of success. Moreover, when users were aware of the choices made by others, popularity was also unpredictable, meaning that on repeating the experiment, the same song could end up with a very different level of popularity.

Although Salganik et al.'s study was limited to a small set of songs created by unknown bands, its conclusions about inequality and unpredictability of popularity appear to apply to cultural artifacts in general and social media production in particular. While this would appear to prohibit prediction of popularity, we argue that understanding how the collective behavior of Web users emerges from the decisions made by interconnected individuals allows us to predict the popularity of items from the users' early reaction to them. As in previous works [15, 16, 22], we use a stochastic modeling framework to mathematically describe the social dynamics of Web users. 
This framework represents each user and each submitted item as a stochastic process with a few states, e.g., a simple Markov processes whose future state depends only on its present state and the input it receives. We used this approach to study collective user activity on the social news aggregator Digg. We produced a model that partially explains - and predicts [27] - the social voting patterns on Digg and related these aggregate behaviors to the ways Digg enables users to discover new content. While this model included social influence, i.e., the increased visibility of stories to a user's neighbors in the social network, it did not address the commonality of users' interests indicated by links. This phenomenon, known as homophily, is a key aspect of social networks. In this paper we describe a new extension to the model that accounts for systematic variations of interests within and outside of the network. We make further changes to the model to more closely match it to web site behavior. First, the new model's state transition rates account for the daily variation in user activity [35], thereby focusing on variations of votes on individual stories compared to the average activity rate on the site. Second, we account for the variation in number of votes a story receives before it is promoted, which the prior model ignored.

By separating the impact of story quality and social influence on the popularity of stories on Digg, a stochastic model of social dynamics enables two novel applications: (1) estimating inherent story quality from the evolution of its observed popularity, and (2) predicting its eventual popularity based on the early reaction of users to the story. Specifically, to predict how popular a story will become, we can use the early votes, even those cast before the story is promoted, to estimate how interesting it is to voters. With this estimate, the model then determines, on average, the story's subsequent evolution. We study these claims empirically on a sample of stories retrieved from Digg. We show that by adjusting for the differing interests among voters, the new model improves predictions of popularity from early reactions of users.

The paper is organized as follows. In Section II we describe details of the social news aggregator Digg, which provides an empirical foundation and a data set for investigating the utility of stochastic models on the prediction task. Section III presents an overview of the stochastic modeling framework. In Section IV] we apply the framework to study dynamics of social voting on Digg. We review an existing model of social dynamics of Digg and show that it explains many of the empirically observed features of aggregate behavior of voters on that site. In Section $\mathrm{V}$ we extend this model to include variations in story interest to users. Then, in Section VI we show how the model can predict eventual popularity of newly submitted stories on Digg.

\section{SOCIAL NEWS PORTAL DIGG}

With over 3 million registered users, the social news aggregator Digg is one of the more popular news portals on the Web. Digg allows users to submit and rate news stories by voting on, or 'digging', them. There are many new submissions every minute, over 16,000 a day. Every day Digg picks about a hundred stories that it believes will be most interesting to the community and promotes them to the front page. Although the exact promotion mechanism is kept secret and changes occasionally, it appears to take into account the number of votes the story receives and how rapidly it receives them. Digg's success is fueled in large part by the emergent front page, which is created by the collective decision of its many users.

While the life cycle of each story may be drastically different from others, its basic elements are the same. These are specified by Digg's user interface, which defines how users can post or discover new stories and interact with other users. A model of social dynamics has to take these elements into account when describing the evolution of story popularity.

\section{A. User interface}

A newly submitted story goes on the upcoming stories list, where it remains for a period of time, typically 24 hours, or until it is promoted to the front page, whichever comes first. The default view shows newly submitted stories as a chronologically ordered list, with the most recently submitted story at the top of the list, 15 stories to a page. To see older stories, a user must navigate to page 2, 3, etc. of the upcoming stories list. Promoted stories (Digg calls them 'popular') are also displayed as a chronologically ordered list on the front pages, 15 stories to a page, with the most recently promoted story at the top of the list. To see older promoted stories, user must navigate to page 2, 3, etc. of the front page. Figure II A shows a screenshot of a Digg front page. Users vote for the stories they like by 'digging' them. The yellow badge to the left of each story shows its current popularity.

Digg also allows users to designate friends and track their activities, i.e., see the stories friends recently submitted or voted for. The friends interface is available through the "Friends' Activity" link at the top of any Digg web page (see, for example, Fig. (IIA). The friend relationship is asymmetric. When user $A$ lists user $B$ as a friend, $A$ can watch the activities of $B$ but not vice versa. We call $A$ the fan of $B$. A newly submitted story is visible in the upcoming stories list, as well as to submitter's fans through the friends interface. With each vote, a story becomes visible to the voter's fans through the friends interface, which shows the newly submitted stories that user's friends voted for.

In addition to these interfaces, Digg also allows users to view the most popular stories from the previous day, week, month, or year. Digg also implements a social filtering feature which recommends stories, including upcoming stories, that were liked by users with a similar voting history. This interface, however, was not available at the time the data for our study was collected and hence is not part of the stochastic models described in this paper. Thus we examine a period of time where Digg had a relatively simple user interface, which simplifies the stochastic models. This choice allow us to focus on evaluating the stochastic model approach, particularly the empirical question of whether averaging is useful in the social media setting in spite of long-tail distributions, which 


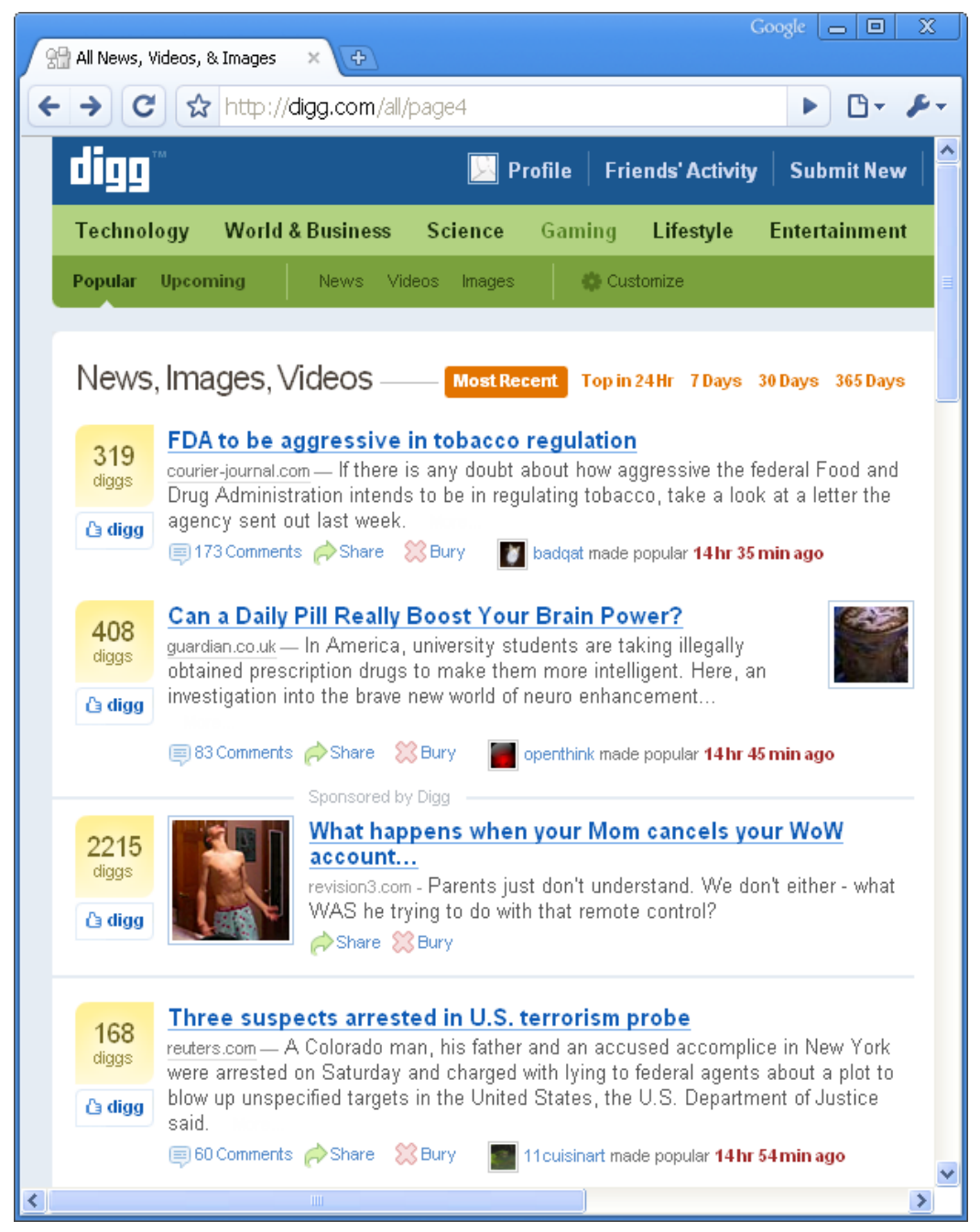

FIG. 1: Screenshot of the front page of the social news aggregator Digg.

contrasts with the narrow distributions found in most statistical physics settings.

\section{B. Dynamics of popularity}

While a story is in the upcoming stories list, it accrues votes slowly. If the story is promoted to the front page, it accumulates votes at a much faster pace. Figure 2(a) shows evolution of the number of votes for two stories submitted in June 2006. The point where the slope abruptly increases corresponds to promotion to the front page. The vast majority of stories are never promoted and, therefore, never experience the sharp rise in the number of votes that accompanies being featured on the front page. As the story ages, accumulation of new votes slows down [38], and after a few days the total number of votes received by a story saturates to some value. This value, which we also call the final number of votes, gives a measure of the story's success or popularity.

Popularity varies widely from story to story. Figure 2(b) shows the distribution of the final number of votes received by front page stories that were submitted over a period of about two days in June 2006. The distribution is characteristic of 'inequality of popularity', since a handful of stories become very popular, accumulating thousands of votes, while most others can only muster a few hundred votes. This distribution applies to front page stories only. Stories that are never promoted to the front page receive very few votes, in many cases just a single vote from the submitter. Such distributions are also called 'long tailed' distributions. This means that in systems displaying such distributions extreme events, e.g., a story receiving many thousands of votes, occur much more frequently than would be expected if the underlying processes were Poisson or Gaussian in nature.

The long tail is a ubiquitous feature [3] of human activity. It is present in inequality of popularity of cultural artifacts, such as books and music albums [34], and also manifests itself in a variety of online behaviors, including tagging, where a few documents are tagged much more frequently than others, collaborative editing on wikis [21], and general social me- 


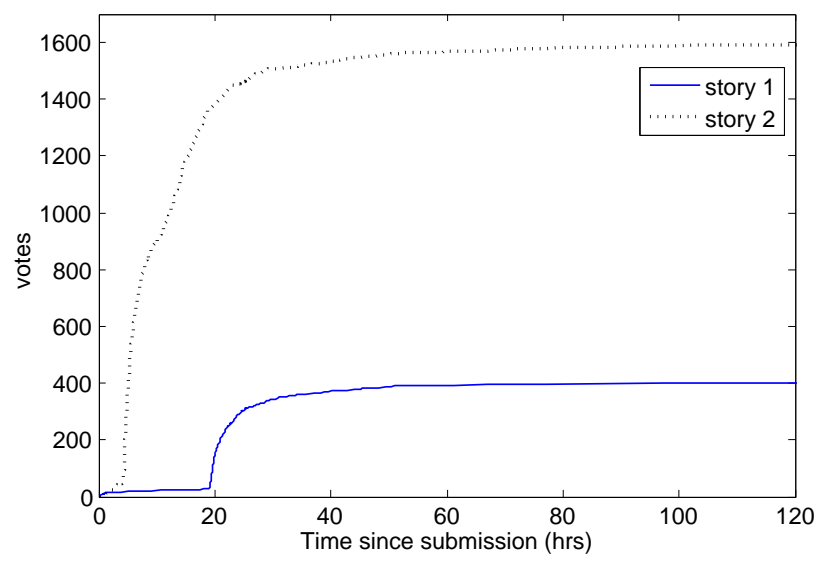

(a)

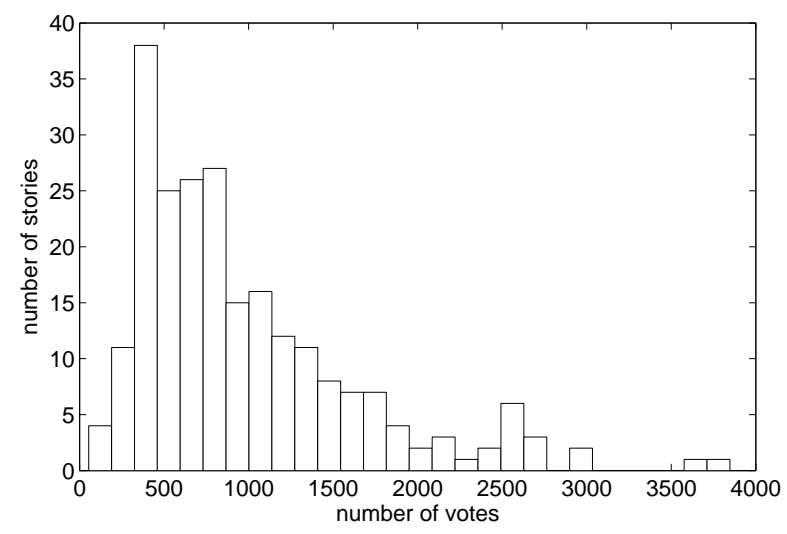

(b)

FIG. 2: Dynamics of social voting. (a) Evolution of the number of votes received by two front page stories in June 2006. (b) Distribution of popularity of 201 front page stories submitted in June 2006.

dia usage [37]. The same distribution of popularity was also observed in a sample of more than 30,000 stories promoted to Digg's front page over the course of a year [38].

While unpredictability of popularity is more difficult to verify than in the controlled experiments of Salganik et al., it is reasonable to assume that a similar set of stories submitted to Digg on another day will end with radically different numbers of votes. In other words, while the distribution of the final number of votes these stories receive will look similar to the distribution in Figure 2 (b), the number of votes received by individual stories will be very different in the two realizations.

\section{Data collection}

We collected data for the study by scraping Digg's Web pages in May and June 2006. The May data set consists of stories that were submitted to Digg May 25-27, 2006. We followed these stories by periodically scraping Digg to determine the number of votes stories received as a function of the time since their submission. We collected at least 4 such observations for each of 2152 stories, submitted by 1212 distinct users. Of these stories, 510, by 239 distinct users, were promoted to the front page. We followed the promoted stories over a period of several days, recording the number of votes the stories received. This May data set also records the location of the stories on the upcoming and front pages as a function of time.

The June data set consists of 201 stories promoted to the front page between June 27 and 30, 2006. For each story, we collected the names of its first 216 voters.

We focus our data collection on the early stages of story evolution - from submission until shortly after promotion. The reason for this is that the Digg social network has a much larger effect on upcoming than front page stories due to the much more rapid addition of stories to the upcoming list. This large influx of stories makes it difficult for users to find a new story before it becomes hidden by the arrival of more stories. In this case, enhanced visibility via the network for fans of the

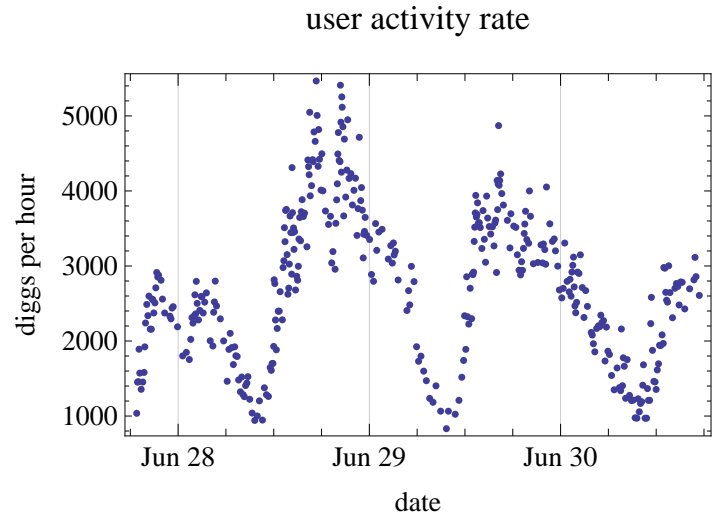

FIG. 3: Voting rate (diggs per hour) on front page stories at the end of June 2006. The indicated dates are the start of each day (0:00 GMT). The minimum in daily activity is around 9am GMT. Each point is the average voting rate for 100 successive votes.

submitter or early voters is particularly important, and a model of social dynamics has to account for it. In light of these observations, and for speeding up data collection, we focus on the early votes for stories.

Activity on Digg varies considerably over the course of a day, as seen in Fig. 3. Adjusting times by the cumulative activity on the site accounts for this variation and improves predictions [35]. We define the "Digg time" between two events (e.g., votes on a story) as the total number of votes on front page stories during the time between those events. In our data set, there are on average about 2500 such votes per hour, with a range of about a factor of 4 in this rate during the course of a day. This behavior is similar to that seen in an extensive study of front page activity in 2007 [35], and as in that study we scale the measure by defining a "Digg hour" to be the average number of front page votes in an hour, i.e., 2500 for our data set. We evaluate the consequence of this variability by contrasting a model based on real time (in Sec. IVP with one based on Digg time (in Sec. V). 
In addition to voter activity, we also extracted a snapshot of the social network of the top-ranked 1020 Digg users as of June 2006. This data contained the names of each user's friends and fans. Since the original network did not contain information about all the voters in our data, we augmented it in February 2008 by extracting names of friends of about 15, 000 additional users. Many of these users added friends between June 2006 and February 2008. Although Digg does not provide the time a new link was created, it lists the links in reverse chronological order and gives the date the friend joined Digg. By eliminating friends who joined Digg after June 30, 2006, we were able to reconstruct the fan links for all voters in our data. This data allows us to identify, for each vote, whether the user was a fan of any prior voter on that story, in which case the story would have appeared in the friends interface for that user.

Votes by fans account for $6 \%$ of the votes in the June data set and about $3 \%$ of the front page votes.

The data sets used in this and previous works were collected before Digg's API was introduced. Scraping Web pages to extract data had several issues. First, data had to be manually cleaned to ensure consistency. Second, since vote time stamps were not available on the Web page, we had to supplement June 2006 data by using the Digg API in October 2009 to obtain the time of each vote, the final number of votes the story received, and the time of promotion. In the intervening time, however, some of the users had deleted their accounts. Since we could not easily resolve the time of the vote of an inactive user, we had to delete these users from the voters list. We believe that the small fraction of data lost in this manner (less than $8 \%$ of the data) does not adversely affect the modeling study. However, in the future we plan to repeat the study on a much cleaner data set obtained through Digg API.

\section{STOCHASTIC MODELS OF SOCIAL DYNAMICS}

Rather than account for the inherent variability of individuals, stochastic models focus on describing the macroscopic, or aggregate, behavior of the system, which can be described by average quantities. In the context of Digg, such quantities include average rate at which users post new stories and vote on existing stories. Such macroscopic descriptions often have a simple form and are analytically tractable. Stochastic models do not reproduce the results of a single observation rather, they describe the 'typical' behavior. These models are analogous to the approach used in statistical physics, demographics and macroeconomics where the focus is on relations among aggregate quantities, such as volume and pressure of a gas, population of a country and immigration, or interest rates and employment.

We represent each individual entity, whether a user or a story, as a stochastic process with a small number of states. This abstraction captures much of the individual complexity and environmental variability by casting user's decisions as inducing probabilistic transitions between states. While this modeling framework applies to stochastic processes of varying complexity, for simplicity, we focus on simple processes that obey the Markov property, namely, a user whose future state depends only on her present state and the input she receives. A Markov process can be succinctly captured by a state diagram showing the possible states of the user and conditions for transition between those states.

We assume that all users have the same set of states, and that transitions between states depend only on the state and not the individual user. That is, the state captures the key relevant properties determining subsequent user actions. Then, the aggregate state of the system can be described simply by the number of individuals in each state at a given time. That is, the system configuration at this time is defined by the occupation vector: $\vec{n}=\left(n_{1}, n_{2}, \ldots\right)$ where $n_{k}$ is the number of individuals in state $k$. For example, in the context of a given story on Digg, one of the states for a user could be "has voted for the story". The component of the occupation vector corresponding to this state is the number of users who have voted for this story, without regard for which particular users those are.

The next step in developing the stochastic model is to summarize the variation within the collection of histories with a probabilistic description. That is, we characterize the possible occupation vectors by the probability, $P(\vec{n}, t)$, the system is in configuration $\vec{n}$ at time $t$. The evolution of $P(\vec{n}, t)$, governed by the Stochastic Master Equation [19], is almost always too complex to be analytically tractable. Fortunately we can simplify the problem by working with the average occupation number, whose evolution is given by the Rate Equation

$$
\frac{d\left\langle n_{k}\right\rangle}{d t}=\sum_{j} w_{j k}(\langle\vec{n}\rangle)\left\langle n_{j}\right\rangle-\left\langle n_{k}\right\rangle \sum_{j} w_{k j}(\langle\vec{n}\rangle)
$$

where $\left\langle n_{k}\right\rangle$ denotes the average number of users in state $k$ at time $t$, i.e., $\sum_{\vec{n}} n_{k} P(\vec{n}, t)$ and $w_{j k}(\langle\vec{n}\rangle)$ is the transition rate from configuration $j$ to configuration $k$ when the occupation vector is $\langle\vec{n}\rangle$.

Using the average of the occupation vector in the transition rates is a common simplifying technique for stochastic models. A sufficient condition for the accuracy of this approximation is that variations around the average are relatively small. In many stochastic models of systems with large numbers of components, variations are indeed small due to many independent interactions among the components. More elaborate versions of the stochastic approach give improved approximations when variations are not small, particularly due to correlated interactions [32]. User behavior on the web, however, often involves distributions with long tails, whose typical behaviors differ significantly from the average [5, 37]. In this case we have no guarantee that the averaged approximation is adequate. Instead we must test its accuracy for particular aggregate behaviors by comparing model predictions with observations of actual behavior, as we report below.

In the Rate Equation, occupation number $n_{k}$ increases due to users' transitions from other states to state $k$, and decreases due to transitions from the state $k$ to other states. The equations can be easily written down from the user state diagram. Each state corresponds to a dynamic variable in the mathematical model - the average number of users in that state 


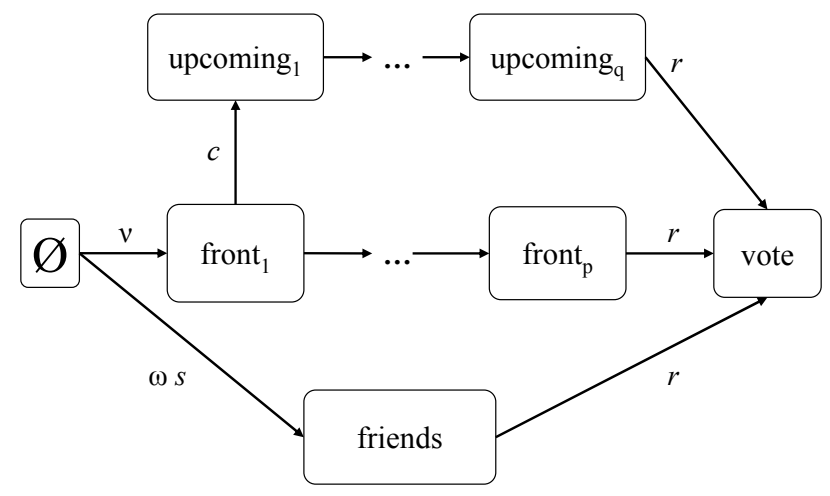

FIG. 4: State diagram of user behavior for a single story. A user starts in the $\emptyset$ state at the left, may find the story through one of the three interfaces and may then vote on it. At a given time, the story is located on a particular page of either the upcoming or front page lists, not both. This diagram shows votes for a story on either page $p$ of the front pages or page $q$ of the upcoming pages. Only fans of previous voters can see the story through the friends interface. Users in the friends, front or upcoming states may choose to leave Digg, thereby returning to the $\emptyset$ state (with those transitions not shown in the figure). Users reaching the "vote" state remain there indefinitely and can not vote on the story again. Parameters next to the arrows characterize state transitions.

- and it is coupled to other variables via transitions between states. Every transition must be accounted for by a term in the equation, with transition rates specified by the details of the interactions between users.

In summary, the stochastic modeling framework requires specifying the aggregate states of interest for describing the system and how individual user behaviors create transitions among these states. The modeling approach is best suited to cases where the users' decisions are mainly determined by a few characteristics of the user and the information they have about the system. These system states and transitions give the rate equations. Solutions to these equations then give estimates of how aggregate behavior varies in time and depends on the characteristics of the users involved.

\section{A MODEL OF SOCIAL DYNAMICS OF DIGG}

Underlying a stochastic model of social dynamics is a behavioral model of an individual Web user. The behavioral model takes into account the choices a Web site's user interface allows users. Detailed data about human activity that can be collected from social media sites such as Digg allow us to parameterize the models and test them by comparing their predictions to the observed collective dynamics.

An earlier study of social dynamics of Digg [15] used a simple behavioral model that viewed each Digg user as a stochastic Markov process, whose state diagram with respect to a single story is shown in Fig 4 . According to this model, a user visiting Digg can choose to browse the front pages to see the recently promoted stories, upcoming stories pages for the re- cently submitted stories, or use the friends interface to see the stories her friends have recently submitted or voted for. She can select a story to read from one of these pages and, if she considers it interesting, vote for it. The user's environment, the stories she is seeing, changes in time due to the actions of all the users.

We characterize the changing state of a story by three values: the number of votes, $N_{\text {vote }}(t)$, the story has received by time $t$ after it was submitted to Digg, the list the story is in at time $t$ (upcoming or frontpage) and its location within that list, which we denote by $q$ and $p$ for upcoming and front page lists, respectively.

With Fig. 4 as a modeling blueprint, we relate the users' choices to the changes in the state of a single story. In terms of the general rate equation (Eq. 1 ), the occupancy vector $\vec{n}$ describing the aggregate user behavior at a given time has the following components: the number of users who see a story via one of the front pages, one of the upcoming pages, through the friends pages, and number of users who vote for a story, $N_{\text {vote }}$. Since we are interested in the number of users who reach the vote state, we do not need a separate equation for each state in Fig. 4. at a given time, a particular story has a unique location on the upcoming or front page lists. Thus, for simplicity, we can group the separate states for each list in Fig. 4, and consider just the combined transition for a user to reach the page containing the story at the time she visits Digg. These combined transition rates depend on the location of the story in the list, i.e., the value of $q$ or $p$ for the story. With this grouping of user states, the rate equation for $N_{\text {vote }}(t)$ is:

$$
\frac{d N_{\text {vote }}(t)}{d t}=r\left(\nu_{\mathrm{f}}(t)+\nu_{\mathrm{u}}(t)+\nu_{\text {friends }}(t)\right)
$$

where $r$ measures how interesting the story is, i.e., the probability a user seeing the story will vote on it, and $\nu_{\mathrm{f}}, \nu_{\mathrm{u}}$ and $\nu_{\text {friends }}$ are the rates at which users find the story via one of the front or upcoming pages, and through the friends interface, respectively.

In this model, the transition rates appearing in the rate equation depend on the time $t$ but not on the occupation vector. Nevertheless, the model could be generalized to include such a dependence if, for example, a user currently viewing an interesting story not only votes on it but explicitly encourages people they know to view the story as well.

\section{A. Story Visibility}

Before we can solve Eq. 2, we must model the rates at which users find the story through the various Digg interfaces. These rates depend on the story's location in the list. The parameters of these models depend on user behaviors that are not readily measurable. Instead, we estimate them using data collected from Digg, as described below.

a. Visibility by position in list A story's visibility on the front page or upcoming stories lists decreases as recently added stories push it further down the list. The stories are shown in groups: the first page of each list displays the 15 most recent stories, page 2 the next 15 stories, and so on. 
We lack data on how many Digg visitors proceed to page 2, 3 and so on in each list. However, when presented with lists over multiple pages on a web site, successively smaller fractions of users visit later pages in the list. One model of users following links through a web site considers users estimating the value of continuing at the site, and leaving when that value becomes negative [17]. This model leads to an inverse Gaussian distribution of the number of pages $m$ a user visits before leaving the web site,

$$
e^{-\frac{\lambda(m-\mu)^{2}}{2 m \mu^{2}}} \sqrt{\frac{\lambda}{2 \pi m^{3}}}
$$

with mean $\mu$ and variance $\mu^{3} / \lambda$. This distribution matches empirical observations in several web settings [17]. When the variance is small, for intermediate values of $m$ this distribution approximately follows a power law, with the fraction of users leaving after viewing $m$ pages decreasing as $m^{-3 / 2}$.

To model the visibility of a story on the $m^{\text {th }}$ front or upcoming page, the relevant distribution is the fraction of users who visit at least $m$ pages, i.e., the upper cumulative distribution of Eq. 3 For $m>1$, this fraction is

$$
f_{\text {page }}(m)=\frac{1}{2}\left(F_{m}(-\mu)-e^{2 \lambda / \mu} F_{m}(\mu)\right)
$$

where $F_{m}(x)=\operatorname{erfc}\left(\alpha_{m}(m-1+x) / \mu\right)$, erfc is the complementary error function, and $\alpha_{m}=\sqrt{\lambda /(2(m-1))}$. For $m=1, f_{\text {page }}(1)=1$.

The visibility of stories decreases in two distinct ways when a new story arrives. First, a story moves down the list on its current page. Second, a story at the $15^{\text {th }}$ position moves to the top of the next page. For simplicity, we model these processes as decreasing visibility, i.e., the value of $f_{\text {page }}(m)$, through $m$ taking on fractional values within a page, i.e., $m=1.5$ denotes the position of a story half way down the list on the first page. This model is likely to somewhat overestimate the loss of visibility for stories among the first few of the 15 items on a given page since the top several stories are visible without requiring the user to scroll down the page.

b. List position of a story Fig. 5(a) shows how the page number of a story on the two lists changes in time for three randomly chosen stories from our data set. The behavior is close to linear when averaging over the daily activity variation (shown in Fig. 3). For simplicity in this model, we ignore this variation and take a story's page number on the upcoming page $q$ and the front page $p$ at time $t$ to be [15]

$$
\begin{aligned}
& p(t)=k_{\mathrm{f}}\left(t-T_{\text {promotion }}\right)+1 \\
& q(t)=k_{\mathrm{u}} t+1
\end{aligned}
$$

where $T_{\text {promotion }}$ is the time the story is promoted to the front page (or $\infty$ if the story is never promoted) and the slopes are given in Table I For a given story, $p(t)$ is only defined for times $t \geq T_{\text {promotion }}$ and $q(t)$ for $t<T_{\text {promotion. Since each }}$ page holds 15 stories, these rates are $1 / 15^{\text {th }}$ the submission and promotion rates, respectively.

c. Front page and upcoming stories lists Digg prominently shows the stories on the front page. The upcoming stories list is less popular than the front page. We model this fact by assuming a fraction $c<1$ of Digg visitors proceed to the upcoming stories pages.

We use a simple threshold to model how a story is promoted to the front page. Initially the story is visible on the upcoming stories pages. If and when the number of votes a story receives exceeds a promotion threshold $h$, the story moves to the front page. This threshold model approximates Digg's promotion algorithm as of May 2006, since in our data set we did not see any front page stories with fewer than 44 votes, nor did we see any upcoming stories with more than 42 votes. We take $h=40$ as an approximation to the promotion algorithm.

d. Friends interface The friends interface allows the user to see the stories her friends have (i) submitted, (ii) voted for, and (iii) commented on in the preceding 48 hours. Although users can take advantage of all these features, we only consider the first two. These uses of the friends interface are similar to the functionality offered by other social media sites: e.g., Flickr allows users to see the latest images his friends uploaded, as well as the images a friend liked.

The fans of the story's submitter can find the story via the friends interface. As additional people vote on the story, their fans can also see the story. We model this with $s(t)$, the number of fans of voters on the story by time $t$ who have not yet seen the story. Although the number of fans is highly variable, the average number of additional fans from an extra vote when the story has $N_{\text {vote }}$ votes is approximately

$$
\Delta s=a N_{\mathrm{vote}}^{-b}
$$

where $a=51$ and $b=0.62$, as illustrated in Fig. 5(b), showing the fit to the increment in average number of fans per vote over groups of 5 votes as given in the data. Thus early voters on a story tend to have more new fans (i.e., fans who are not also fans of earlier voters) than later voters.

The model can incorporate any distribution for the times fans visit Digg. We suppose these users visit Digg daily, and since they are likely to be geographically distributed across all time zones, the rate fans discover the story is distributed throughout the day. A simple model of this behavior takes fans arriving at the friends page independently at a rate $\omega$. As fans read the story, the number of potential voters gets smaller, i.e., $s$ decreases at a rate $\omega s$, corresponding to the rate fans find the story through the friends interface, $\nu_{\text {friends. }}$. We neglect additional reduction in $s$ from fans finding the story without using the friends interface.

Combining the growth in the number of available fans and its decrease as fans return to Digg gives

$$
\frac{d s}{d t}=-\omega s+a N_{\mathrm{vote}}^{-b} \frac{d N_{\mathrm{vote}}}{d t}
$$

with initial value $s(0)$ equal to the number of fans of the story's submitter, $S$. This model of the friends interface treats the pool of fans uniformly. That is we assume no difference in behavior, on average, for fans of the story's submitter vs. fans of other voters. 


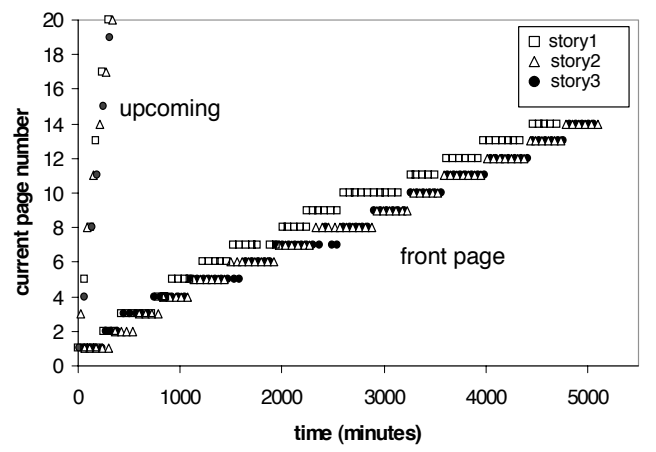

(a)

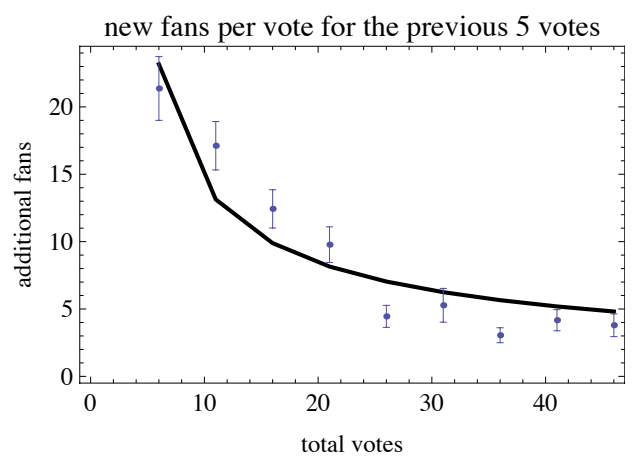

(b)

FIG. 5: (a) Current page number on the upcoming and front pages vs. time for three different stories. Time is measured from when the story first appeared on each page, i.e., time it was submitted or promoted, for the upcoming and front page points, respectively. (b) Increase in the number of distinct users who can see the story through the friends interface with each group of five new votes for the first 46 users to vote on a story. The points are mean values for 195 stories, including those shown in (a), and the curve is based on Eq. 7 The error bars indicate the standard error of the estimated means.

\begin{tabular}{l|l}
\hline parameter & value \\
\hline rate general users come to Digg & $\nu=600$ users $/ \mathrm{hr}$ \\
fraction viewing upcoming pages & $c=0.3$ \\
rate a voters' fans come to Digg & $\omega=0.12 / \mathrm{hr}$ \\
page view distribution & $\mu=0.6, \lambda=0.6$ \\
fans per new vote & $a=51, b=0.62$ \\
vote promotion threshold & $h=40$ \\
upcoming stories location & $k_{\mathrm{u}}=3.60$ pages $/ \mathrm{hr}$ \\
front page location & $k_{\mathrm{f}}=0.18$ pages $/ \mathrm{hr}$ \\
\hline \multicolumn{2}{c}{ story specific parameters } \\
interestingness & $r$ \\
number of submitter's fans & $S$
\end{tabular}

TABLE I: Model parameters.

In summary, the rates in Eq. 2 2 are [40]:

$$
\begin{aligned}
\nu_{\mathrm{f}} & =\nu f_{\text {page }}(p(t)) \Theta\left(N_{\text {vote }}(t)-h\right) \\
\nu_{\mathrm{u}} & =c \nu f_{\text {page }}(q(t)) \Theta\left(h-N_{\text {vote }}(t)\right) \Theta(24 \mathrm{hr}-t) \\
\nu_{\text {friends }} & =\omega s(t)
\end{aligned}
$$

where $t$ is time since the story's submission and $\nu$ is the rate users visit Digg. The first step function in $\nu_{\mathrm{f}}$ and $\nu_{\mathrm{u}}$ indicates that when a story has fewer votes than required for promotion, it is visible in the upcoming stories pages; and when $N_{\text {vote }}(t)>h$, the story is visible on the front page. The second step function in $\nu_{\mathrm{u}}$ accounts for a story staying in the upcoming list for at most 24 hours. We solve Eq. 2 subject to initial condition $N_{\text {vote }}(0)=1$, because a newly submitted story starts with a single vote, from the submitter.

\section{B. Model Parameters}

The solutions of Eq. 2 show how the number of votes received by a story changes in time. The solutions depend on the model parameters, of which only two parameters - the story's interestingness $r$ and number of fans the submitter has $S$ - change from one story to another. Therefore, we fix values of the remaining parameters as given in Table $[$ ]

As described above, we estimate some of these parameters (such as the growth in list location, promotion threshold and fans per new vote) directly from the data. The remaining parameters are not directly given by our data set (e.g., how often users view the upcoming pages) and instead we estimate them based on the model predictions. The small number of stories in our data set, as well as the approximations made in the model, do not give strong constraints on these parameters. We selected one set of values giving a reasonable match to our observations. For example, the rate fans visit Digg and view stories via the friend's interface, given by $\omega$ in Table 1 . has $90 \%$ of the fans of a new voter returning to Digg within the next 19 hours. As another example of interpreting these parameter values, for the page visit distribution the values of $\mu$ and $\lambda$ in Table I correspond to about $1 / 6$ of the users viewing more than just the first page. These parameters could in principle be measured independently from aggregate behavior with more detailed information on user behavior. Measuring these values for users of Digg, or other similar web sites, could improve the choice of model parameters.

\section{Results}

The model describes the behavior of all stories, whether or not they are promoted to the front page. To illustrate the model results, we consider stories promoted to the front page. Fig. 6 shows the behavior of six stories. For each story, $S$ is the number of fans of the story's submitter, available from 


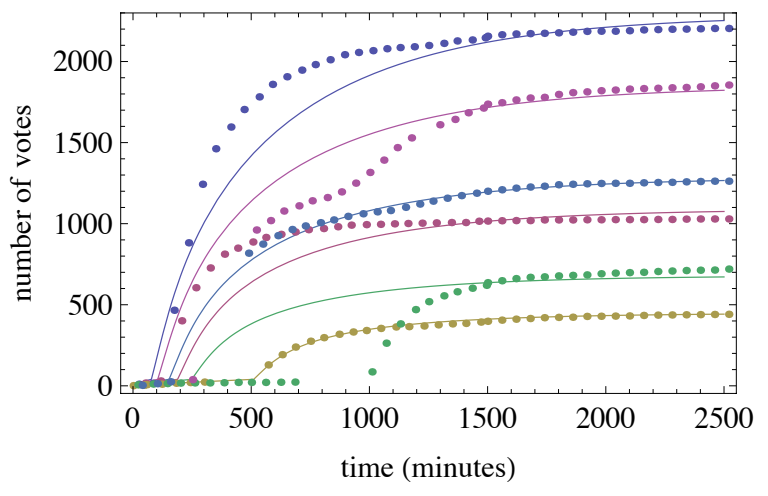

FIG. 6: Evolution of the number of votes received by six stories compared with model solution.

\begin{tabular}{ccc}
$\mathrm{S}$ & $\mathrm{r}$ & final votes \\
\hline 5 & 0.51 & 2229 \\
5 & 0.44 & 1921 \\
40 & 0.32 & 1297 \\
40 & 0.28 & 1039 \\
160 & 0.19 & 740 \\
100 & 0.13 & 458
\end{tabular}

TABLE II: Parameters for the example stories, listed in decreasing order of total votes received by the story and hence corresponding to the curves in Fig. 6 from top to bottom.

our data, and $r$ is estimated to minimize the root-mean-square (RMS) difference between the observed votes and the model predictions. Table II lists these values.

Overall there is qualitative agreement between the data and the model, indicating that the features of the Digg user interface we considered can explain the patterns of collective voting. Specifically, the model reproduces three generic behaviors of Digg stories: (1) slow initial growth in votes of upcoming stories; (2) more interesting stories (higher $r$ ) are promoted to the front page (inflection point in the curve) faster and receive more votes than less interesting stories; (3) however, as first described in [23], better connected users (high $S$ ) are more successful in getting their less interesting stories (lower $r$ ) promoted to the front page than poorly-connected users. These observations highlight a benefit of the stochastic approach: identifying simple models of user behavior that are sufficient to produce the aggregate properties of interest.

The only significant difference between the data and the model is visible in the lower two lines of Fig. 6 In the data, a story posted by the user with $S=100$ is promoted before the story posted by the user with $S=160$, but saturates at smaller value of votes than the latter story. In the model, the story with larger $r$ is promoted first and gets more votes.

Thus while the stochastic model is primarily intended to describe typical story behavior, we see it gives a reasonable match to the actual vote history of individual stories. Nevertheless, there are some cases where individual stories dif-

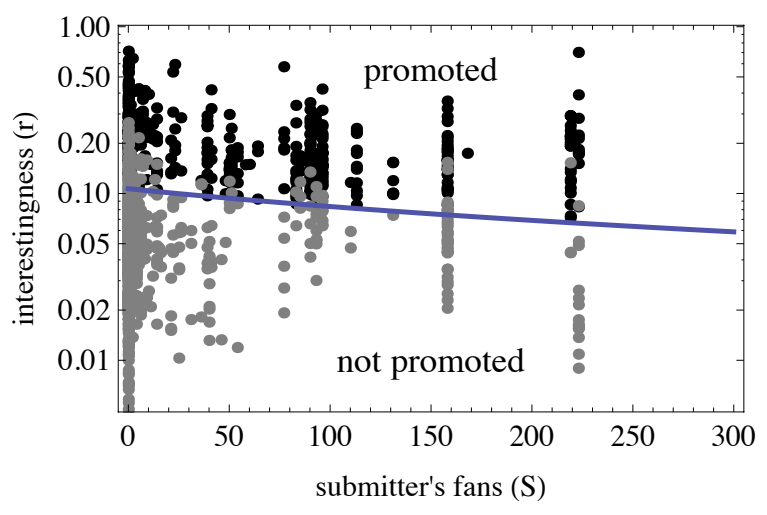

FIG. 7: Story promotion as a function of $S$ and $r$. The $r$ values are shown on a logarithmic scale. The model predicts stories above the curve are promoted to the front page. The points show the $S$ and $r$ values for the stories in our data set: black and gray for stories promoted or not, respectively.

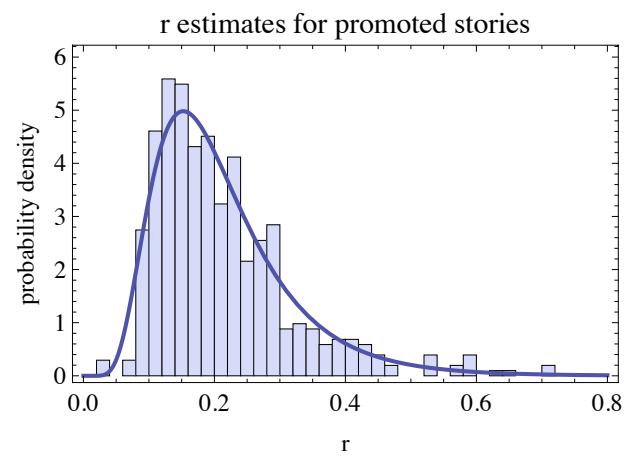

FIG. 8: Distribution of interestingness (i.e., $r$ values) for the promoted stories in our data set compared with the best fit lognormal distribution.

fer considerably from the model, particularly where an early voter happens to have an exceptionally large number of fans, thereby increasing the story's visibility to other users far more than the average number of new fans per vote. This variation, a consequence of the long-tail distributions involved in social media, is considerably larger than seen, for example, in most statistical physics applications of stochastic models. The effect of such large variations is an important issue for addressing the usefulness of the stochastic modeling approach for social media when applied to the behavior of individual stories.

Fig. 7 shows parameters required for a story to reach the front page according to the model, and how that prediction compares to the stories in our data set. The model's prediction of whether a story is promoted is correct for $95 \%$ of the stories in our data set. For promoted stories, the correlation between $S$ and $r$ is -0.13 , which is significantly different from zero ( $p$-value less than $10^{-4}$ by a randomization test). Thus a story submitted by a poorly connected user (small $S$ ) tends to need high interest (large $r$ ) to be promoted to the front page [23].

Figure 8 shows the estimated $r$ values for the 510 promoted 


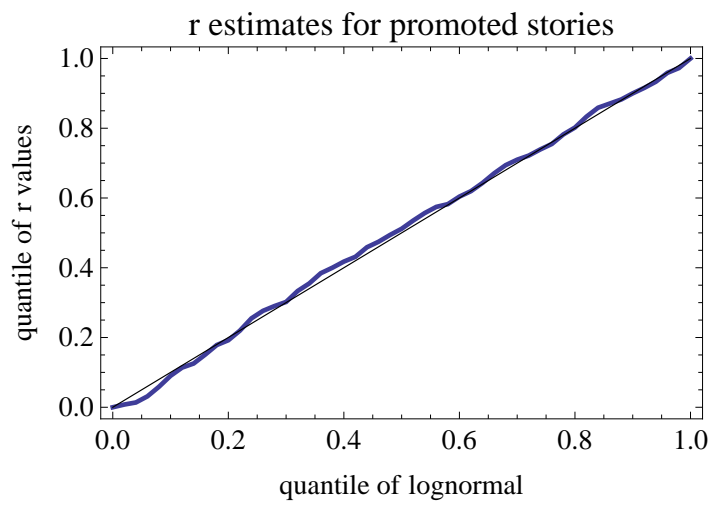

FIG. 9: Quantile-quantile plot comparing observed distribution of $r$ values with the lognormal distribution fit (thick curve). For comparison, the thin straight line from 0 to 1 corresponds to a perfect match between the data and the distribution.

stories in our data set have a wide range of interestingness to users. That is, even after accounting for the variation in visibility of the stories, there remains a significant range in how well stories appeal to users. Specifically, Fig. 9 shows these $r$ values fit well to a lognormal distribution

$$
P_{\text {lognormal }}(\mu, \sigma ; r)=\frac{1}{\sqrt{2 \pi} r \sigma} \exp \left(-\frac{(\mu-\log (r))^{2}}{2 \sigma^{2}}\right)
$$

where parameters $\mu$ and $\sigma$ are the mean and standard deviation of $\log (r)$. For the distribution of interestingness values, the maximum likelihood estimates of the mean and standard deviation of $\log (r)$ equal to $-1.67 \pm 0.04$ and $0.47 \pm 0.03$, respectively, with the ranges giving the $95 \%$ confidence intervals. A randomization test based on the Kolmogorov-Smirnov statistic and accounting for the fact that the distribution parameters are determined from the data [8] shows the $r$ values are consistent with this distribution ( $p$-value 0.35). While broad distributions occur in several web sites [37], our model allows factoring out the effect of visibility due to the user interface from the overall distribution of votes. Thus we can identify variation in users' inclination to vote on a story they see.

The simple model described in this section gives a reasonable qualitative account of how user behavior leads to stories' promotion to the front page and the eventual saturation in the number of votes they receive due to their decreasing visibility. In the section below we show how additional properties of the interface and user population can be added to the model for a more accurate analysis of the aggregate behavior. For example, submitter's fans may find the story more interesting than the general Digg audience, corresponding to different $r$ values for these groups of users. In addition, we modeled users coming to Digg independently with uniform rates $\nu$ and $\omega$. In fact, the rates vary systematically over hours and days [35] as shown in Fig. 3, and individual users have a wide range in time between visits [36]. In our model, this variation gives time-dependent values for $\nu$, describing the rate users come to Digg, and $k_{\mathrm{f}}$ and $k_{\mathrm{u}}$, which relate to the rate new stories are posted and promoted.

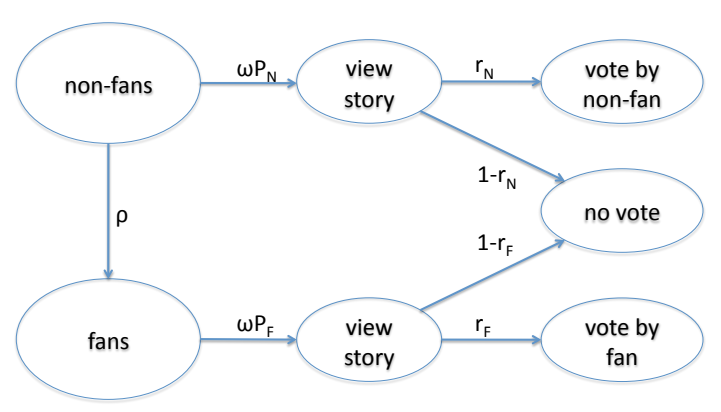

FIG. 10: State diagram for a user. The submitter provides a story's first vote. The initial set of fans consists of the submitter's fans; other users are initially non-fans. Fans and non-fans have different probabilities to see and vote on the story. With each vote, a nonfan user who is a fan of that voter moves into the fans state. This state transition is caused by the votes of other users: a user moving from the non-fans to fans state is not aware of that change until later visiting Digg and seeing the story in the friends interface.

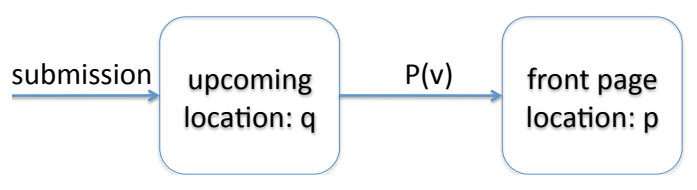

FIG. 11: State diagram for a story. A story starts at the top of the upcoming pages, with location $q=1$. The location increases with each new submission. An upcoming story with $v$ votes is promoted with probability $P(v)$. A promoted story starts at the top of the front pages, with location $p=1$. The location increases as more stories are promoted. A story not promoted within a day is removed (not shown).

The ability of the stochastic approach to incorporate additional details in the user models illustrates its value in providing insights into how aggregate behavior arises from the users, in contrast to models that evaluate regularities in the aggregate behaviors [38]. In particular, user models can help distinguish aggregate behaviors arising from intrinsic properties of the stories (e.g., their interestingness to the user population) from behavior due to the information the web sites provides, such as ratings of other users and how stories are placed in the site, i.e., visibility. Finally, stochastic models have not only explanatory, but also predictive power.

\section{A MODEL OF SOCIAL VOTING WITH NICHE INTERESTS}

To investigate differences among voters with respect to the friends network, we extend the previous stochastic model to distinguish votes from fans and non-fans. The model considers the joint behavior of users and the location of the story on the web site. Fig. 10 shows the user states and the stochastic transitions between them. Stories are on either the upcoming or front pages, as shown in Fig. 11 This leads to a description of the average rates of growth for votes from fans and 
non-fans of prior voters, $v_{F}$ and $v_{N}$, respectively:

$$
\begin{aligned}
\frac{d v_{F}}{d t} & =\omega r_{F} P_{F} F \\
\frac{d v_{N}}{d t} & =\omega r_{N} P_{N} N
\end{aligned}
$$

where $t$ is the Digg time since the story's submission and $\omega$ is the average rate a user visits Digg (measured as a rate per unit Digg time). $v_{N}$ includes the story's submitter. $P_{F}$ and $P_{N}$ denote the story's visibility and $r_{F}$ and $r_{N}$ denote the story's interestingness to users who are fans or not of prior voters, respectively. Visibility depends on the story's state (e.g., whether it has been promoted), as discussed below. Interestingness is the probability a user who sees the story will vote on it. Nominally people become fans of those whose contributions they consider interesting, suggesting fans likely have a systematically higher interest in stories. Our model accounts for this possibility with separate interestingness values for fans and non-fans.

In contrast to the model of Sec. IV] where time $t$ denoted real time since story submission, we now use $t$ to denote the "Digg time" since submission, thereby accounting for the daily variation in activity. Using Digg time reduces the variation in the rate users visit Digg, thereby improving the match to the assumed constant rate $\omega$ used in the model. Moreover, a detailed examination of the page locations of the stories in our data set, shows systematic variation in the time stories spend on each page corresponding to the daily activity variation used to define Digg time. Thus using Digg time improves the accuracy of the linear growth in location given in Eq. (5) and (6).

These voting rates depend on $F(N)$, the numbers of users who have not yet seen the story and who are (are not) fans of prior voters. The quantities change as users see and vote on the story according to

$$
\begin{aligned}
\frac{d F}{d t} & =-\omega P_{F} F+\rho N \frac{d v}{d t} \\
\frac{d N}{d t} & =-\omega P_{N} N-\rho N \frac{d v}{d t}
\end{aligned}
$$

with $v=v_{F}+v_{N}$ the total number of votes the story has received. The quantity $\rho$ is the probability a user who has not yet seen the story and is not a fan of a prior voter is a fan of the most recent voter. For simplicity, we treat this probability as a constant over the voters, thus averaging over the variation due to clustering in the social network and the number of fans a user has. The first term in each of these equations is the rate the users see the story. The second terms arise from the rate the story becomes visible in the friends interface of users who are not fans of previous voters but are fans of the most recent voter.

Initially, the story has one vote (from the submitter) and the submitter has $S$ fans, so $v_{F}(0)=0, v_{N}(0)=1, F=S$ and $N=U-S-1$ where $U$ is the total number of active users at the time the story is submitted. Over time, a story becomes less visible to users as it moves down the upcoming or (if promoted) front page lists, thereby attracting fewer votes and hence fewer new fans of prior voters.

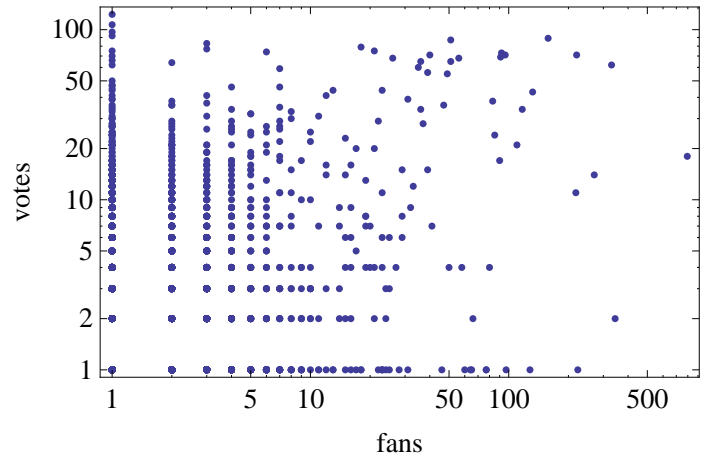

FIG. 12: Comparison of activity (number of votes) and number of fans for each of the 3436 users with at least one vote and one fan.

We use the same visiting rate parameter, $\omega$, for users who are and are not fans of prior voters since there is only a small correlation between voting activity and the number of fans across all the stories in our data set, as illustrated in Fig. 12. Moreover, many highly active users do not participate in the social network at all (i.e., have neither fans nor friends). Among all users, the correlation between number of votes and number fans is 0.15 . More specifically, we assume that with respect to votes on a single story, fans of those voters aren't systematically more likely to visit Digg than other users, such as fans of voters on other stories or users without fans or friends.

\section{A. Story Visibility}

We assume a fan easily sees the story via the friends interface, so $P_{F}=1$, as in the previous model [15]. Users who are not fans of prior voters must find the story on the front or upcoming pages. Thus $P_{N}$ depends on how users navigate through these pages and the story's location at the time the user visits Digg. As with the previous model, we use Eq. 3 to describe this behavior.

e. List position of a story The page number of a story on the upcoming page $q$ and the front page $p$ at time $t$ is given by Eq. (5) and (6), with $t$ now interpreted as Digg time. The slopes, given in Table III, are the same as with the previous model which averaged over the daily variation in activity. Since each page holds 15 stories, these rates are $1 / 15^{\text {th }}$ the story submission and promotion rates, respectively.

Since upcoming stories are less popular than the front page, our model has a fraction $c<1$ of Digg visitors viewing the upcoming stories pages. Combining these effects, we take the visibility of a story at position $p$ in the front page list to be $P_{N}=f_{\text {page }}(p)$, whereas a story at position $q$ in the upcoming page list is $c f_{\text {page }}(q)$ [15].

$f$. Promotion to the front page Promotion to the front page appears to depend mainly on the number of votes the story receives. We model this process by the probability $P(v)$ an upcoming story is promoted after its $v^{t h}$ vote. We take $P(1)=0$, i.e., a story is not promoted just based on the sub- 


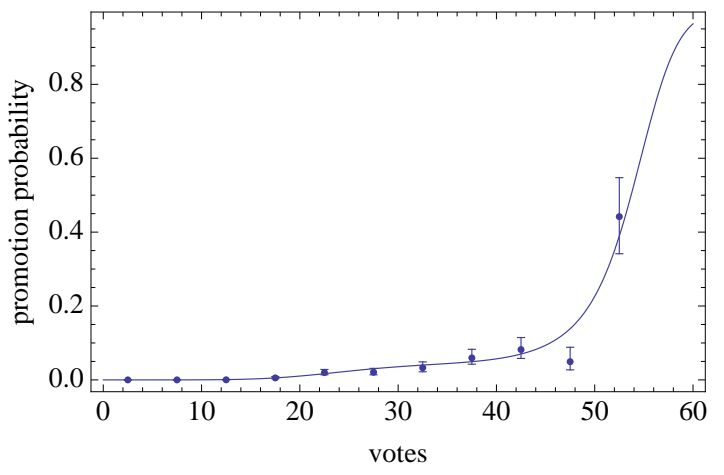

FIG. 13: Probability for promotion before the next vote for an upcoming story as a function of the number of votes. The error bars indicate the $95 \%$ confidence intervals for the estimates. The curve is a logistic fit.

mitter's vote. The probability a story is not promoted by the time it receives $v$ votes is $\prod_{i=1}^{v}(1-P(i))$. Stories not promoted are eventually removed, typically 24 hours after submission.

Based on our data, Fig. 13 shows the probability $P(v)$ an upcoming story is promoted after $v$ votes conditioned on it not having been promoted earlier. We find a significant spread in the number of votes a story has when it is promoted. For predicting whether and when a story will be promoted in our model, we use a logistic regression fit to these values, as shown in the figure. This contrasts with the step function for promotion at 40 votes used in the previous model [15].

g. Friends interface The fans of the story's submitter can find the story via the friends interface. As additional people vote on the story, their fans can also see the story. We model this with $F(t)$, the number of fans of voters on the story by time $t$ who have not yet seen the story. Although the number of fans is highly variable, we use the average number of additional fans from an extra vote, $\rho N$, in Eq. (12).

\section{B. Parameter Estimation}

Since we observe votes, not visits to Digg, there is some ambiguity in the rate $\omega$ and the interestingness values $r_{F}, r_{N}$. For example, a given value of $\omega r_{F}$ could arise from users often visiting Digg but rarely voting on stories, or less frequent visits with a high chance of voting during each visit. This arbitrary scaling does not affect our focus on the relative behavior of fans and non-fans. For definiteness, we pick a specific value for $\omega$ and give interestingness values relative to that choice.

We used the May data to estimate the story location parameters $k_{\mathrm{u}}$ and $k_{\mathrm{f}}$. Their values correspond to 54 and 2.7 stories per hour submitted and promoted, respectively.

\section{Estimating parameters from observed votes}

In our model, story location affects visibility only for nonfan voters since fans of prior voters see the story via the friends interface. Thus we use just the non-fan votes to estimate visibility parameters, via maximum likelihood. Specifically, we use the non-fan votes for 16 stories in the June data set to estimate $c$ and the "law of surfing" parameters $\mu$ and $\lambda$. We then use fan votes for these stories to evaluate the probability a user is a fan of a new voter, $\rho$. Separating votes by the different interfaces by which users find stories provides more precise estimation than the prior model [15].

This estimation involves comparing the observed votes to the voting rate from the model. As described above, the model uses rate equations to determine the average behavior of the number of votes. A simple approach to relate this average to the observed number of votes is to assume the votes from non-fan users form a Poisson process whose expected value is $d v_{N}(t) / d t$, given by Eq. (11). This rate changes with time and depends on the model parameters.

For a Poisson process with a constant rate $v$, the probability to observe $n$ events in time $T$ is the Poisson distribution $e^{-v T}(v T)^{n} / n$ !. This probability depends only on the number of events, not the specific times at which they occur. Thus estimating a constant rate involves maximizing this expression, which gives $v=n / T$, i.e., the maximum-likelihood estimate of the rate for a constant Poisson process is equal to the average rate of the observed events.

In our case, the voting rate changes with time, requiring a generalization of this estimation. Specifically consider a Poisson process with nonnegative rate $v(t)$ which depends on one or more parameters to be estimated. Thus in a small time interval $(t, t+\Delta T)$, the probability for a vote is $v(t) \Delta t$, and this is independent of votes in other time intervals, by the definition of a Poisson process. Suppose we observe $n$ votes at times $0<t_{1}<t_{2}, \ldots<t_{n}<T$ during an observation time interval $(0, T)$. Considering small time intervals $\Delta t$ around each observation, the probability of this observation is

$$
\begin{array}{r}
P\left(\text { no vote in }\left(0, t_{1}\right)\right) v\left(t_{1}\right) \Delta t \quad \times \\
P\left(\text { no vote in }\left(t_{1}, t_{2}\right)\right) v\left(t_{2}\right) \Delta t \quad \times \\
\ldots \\
P\left(\text { no vote in }\left(t_{n-1}, t_{n}\right)\right) v\left(t_{n}\right) \Delta t \quad \times \\
P\left(\text { no vote in }\left(t_{n}, T\right)\right)
\end{array}
$$

The probability for no vote in the interval $(a, b)$ is

$$
\exp \left(-\int_{a}^{b} v(t) d t\right)
$$

Thus the log-likelihood for the observed sequence of votes is

$$
-\int_{0}^{T} v(t) d t+\sum_{i} \log v\left(t_{i}\right)
$$

The maximum-likelihood estimation for parameters determining the rate $v(t)$ is a trade-off between these two terms: attempting to minimize $v(t)$ over the range $(0, T)$ to increase the first term while maximizing the values $v\left(t_{i}\right)$ at the specific times of the observed votes. If $v(t)$ is constant, this likelihood expression simplifies to $-v T+n \log v$ with maximum 
at $v=n / T$ as discussed above for the constant Poisson process. When $v(t)$ varies with time, the maximization selects parameters giving relatively larger $v(t)$ values where the observed votes are clustered in time.

In our case, we combine this log-likelihood expression from the votes on several stories, and maximize the combined expression with respect to the story-independent parameters of the model, with the interestingness parameters determined separately for each story.

\section{Estimating number of active users}

Our model involves a population of "active users" who visit Digg during our sample period. Specifically, the model uses the rate users visit Digg, $\omega U$. We do not observe visits in our data, but can infer the relevant number of active users, $U$, from the heterogeneity in the number of votes by users. The June data set consists of 16283 users who voted at least once during the sample period. Fig. 14 shows the distribution of this activity on front page stories. Most users have little activity during the sample period, suggesting a large fraction of users vote infrequently enough to never have voted during the time of our data sample. This behavior can be characterized by an activity rate for each user. A user with activity rate $\nu$ will, on average, vote on $\nu T$ stories during a sample time $T$. We model the observed votes as arising from a Poisson process whose expected value is $\nu T$ and the heterogeneity arising from a lognormal distribution of user activity rates [16]. This model gives rise to the extended activity distribution while accounting for the discrete nature of the observations. The latter is important for the majority of users who have low activity rates so will vote only a few times, or not at all, during our sample period.

Specifically, for $n_{k}$ users with $k$ votes during the sample period, this mixture of lognormal and Poisson distributions [6, [30] gives the log-likelihood of the observations as

$$
\sum_{k} n_{k} \log P(\mu, \sigma ; k)
$$

where $P(\mu, \sigma ; k)$ is the probability of a Poisson distribution to give $k$ votes when its mean is chosen from a lognormal distribution $P_{\text {lognormal }}$ with parameters $\mu$ and $\sigma$. From Eq. (9),

$$
P(\mu, \sigma ; k)=\frac{1}{\sqrt{2 \pi} \sigma k !} \int_{0}^{\infty} \rho^{k-1} e^{-\frac{(\log (\rho)-\mu)^{2}}{2 \sigma^{2}}-\rho} d \rho
$$

for integer $k \geq 0$. We evaluate this integral numerically. In terms of our model parameters, the value of $\mu$ in this distribution equals $\nu T$.

Since we don't observe the number of users who did not vote during our sample period, i.e., the value of $n_{0}$, we cannot maximize this log-likelihood expression directly. Instead, we use a zero-truncated maximum likelihood estimate [14] to determine the parameters $\mu$ and $\sigma$ for the vote distribution of Fig. 14. Specifically, the fit is to the probability of observing $k$ votes conditioned on observing at least one vote. This conditional distribution is $P(\mu, \sigma ; k) /(1-P(\mu, \sigma ; 0))$ for $k>0$,

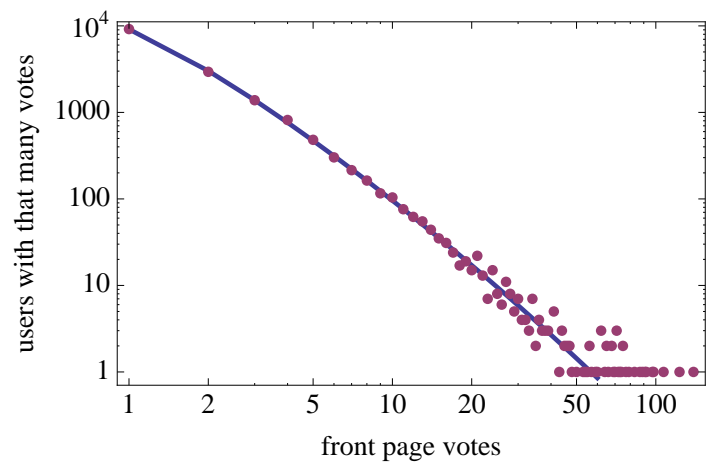

FIG. 14: User activity distribution on logarithmic scales. The curve shows the fit to the model described in the text.

and the corresponding log-likelihood is

$$
\sum_{k>0} n_{k} \log P(\mu, \sigma ; k)-U_{+} \log (1-P(\mu, \sigma ; 0))
$$

where $U_{+}$is the number of users with at least one vote in our sample, i.e., 16283. Maximizing this expression with respect to the distribution's parameters $\mu$ and $\sigma$ gives $\nu T$ lognormally distributed with the mean and standard deviation of $\log (\nu T)$ equal to $-2.06 \pm 0.03$ and $1.82 \pm 0.03$, respectively. With these parameters, $P(\mu, \sigma ; 0)=0.757$, indicating about $3 / 4$ of the users had sufficiently low, but nonzero, activity rate that they did not vote during the sample period. We use this value to estimate $U$, the number of active users during our sample period: $U=U_{+} /(1-P(\mu, \sigma ; 0))$.

Based on this fit, the curve in Fig. 14 shows the expected number of users with each number of votes, i.e., the value of $U P(\mu, \sigma ; k)$ for $k>0$. This is a discrete distribution: the lines between the expected values serve only to distinguish the model fit from the points showing the observed values. A bootstrap test [12] based on the Kolmogorov-Smirnov (KS) statistic shows the vote counts are consistent with this distribution ( $p$-value 0.48 ). This test and the others reported in this paper account for the fact that we fit the distribution parameters to the data [8].

\section{Estimated parameters}

Table III lists the estimated parameters. We estimate $r_{F}$ and $r_{N}$ for each story from its fan and non-fan votes.

The page view distribution seen in this data set indicates users who choose to visit the upcoming pages tend to explore those pages fairly deeply. This contrasts with the more limited exploration, i.e., smaller value of $\mu$, seen in the May data set which included votes well after promotion [15]. This suggests differing levels of perseverance of users who visit the upcoming stories compared to the majority of users who focus on front page stories. Alternatively, there could be other ways non-fan users find content that has already moved far down the list of stories. 


\begin{tabular}{l|l}
\hline parameter & value \\
\hline average rate each user visits Digg & $\omega=0.2 / \mathrm{hr}$ \\
number of active users & $U=70,000$ \\
fraction viewing upcoming pages & $c=0.065$ \\
page view distribution & $\mu=6.3$ \\
& $\lambda=0.14$ \\
probability a user is a voter's fan & $\rho=9.48 \times 10^{-6}$ \\
upcoming stories location & $k_{\mathrm{u}}=3.60$ pages $/ \mathrm{hr}$ \\
front page location & $k_{\mathrm{f}}=0.18$ pages $/ \mathrm{hr}$ \\
\hline
\end{tabular}

story specific parameters

interestingness to fans

interestingness to non-fans

number of submitter's fans
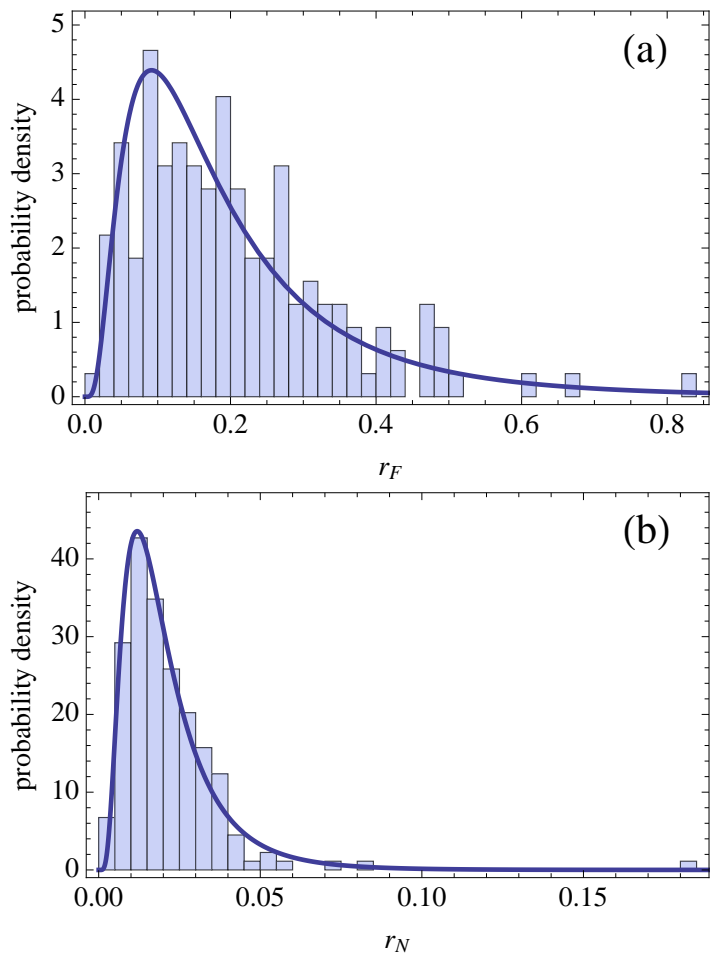

FIG. 16: Distribution of interestingness for (a) fans, and (b) nonfans. The curves are lognormal fits to the values. Note the different ranges for the horizontal scales in the two plots: $r_{F}$ values tend to be significantly larger than $r_{N}$ values.

17 stories have no votes by fans leading to a maximum likelihood estimate $r_{F}=0$, though with a large confidence interval. The remaining values are approximately lognormally distributed with maximum likelihood estimates of the mean and standard deviation of $\log \left(r_{F}\right)$ equal to $-1.8 \pm 0.1$ and $0.75 \pm 0.08$, respectively. The KS statistic indicates the weaker fit, with a $p$-value of 0.04 . Due to the relatively few votes, the discrete nature of the observations likely significantly affects the estimates. For example, a story with no fans among the early votes may reflect a submitter with no fans and a low, but nonzero, interestingness for fans. A subsequent vote by a highly connected user would expose the story to many fans, possibly leading to many votes that the model would miss by assuming $r_{F}=0$. One approach to this difficulty is using the lognormal distributions of $r$ values as priors in the estimation. This procedure somewhat improves performance, as discussed below.

Overall, Fig. 18 shows there is little relation between how interesting a story is to fans and other users: the correlation between $r_{F}$ and $r_{N}$ is -0.11 . A randomization test indicates this small correlation is only marginally significant, with $p$ value 0.05 of arising from uncorrelated values. The relationship between interestingness for fans and other users indicates a considerable variation in how widely stories appeal to the general user community. Specifically, the ratio $r_{F} / r_{N}$ ranges from 0 to 87, with median 9.3. The high values correspond to stories that do not get a large number of votes, indicating they are of significantly more interest to the fans of voters than to

Because there are relatively few votes by fans, we have a

larger variance in estimates of $r_{F}$ than for $r_{N}$. In particular, of $\log \left(r_{N}\right)$ equal to $-4.0 \pm 0.1$ and $0.63 \pm 0.07$, respectively, with the ranges giving the $95 \%$ confidence intervals. A bootstrap test based on the KS statistic shows the $r$ values are consistent with this distribution ( $p$-value 0.1 ). 


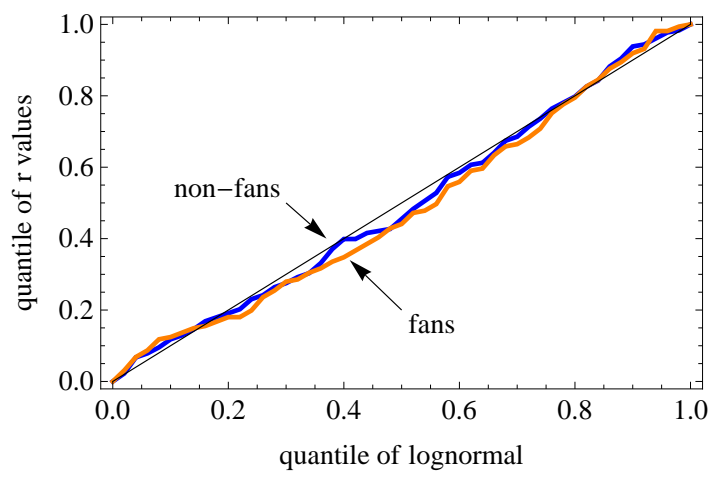

FIG. 17: Quantile-quantile plot comparing the observed distribution for $r_{F}$ (fans) and $r_{N}$ (non-fans) with the corresponding lognormal distribution fits (thick curves). For comparison, the thin straight line from 0 to 1 corresponds to a perfect match between the data and the distribution.

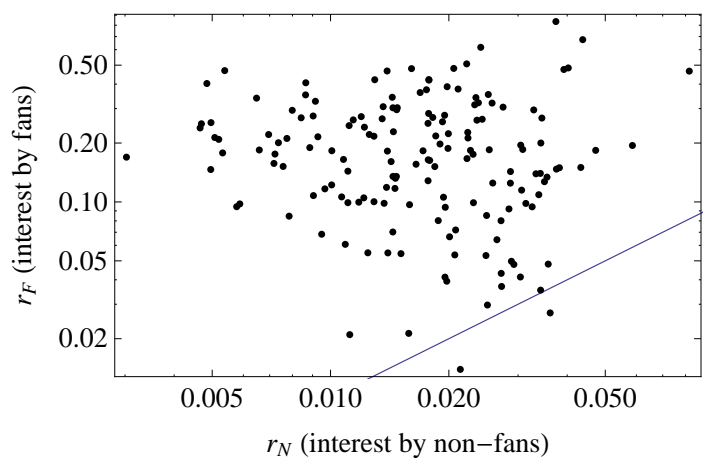

FIG. 18: Log-log plot comparing estimated interestingness to fans $\left(r_{F}\right)$ and non-fans $\left(r_{N}\right)$ for 161 promoted stories with votes from fans (so the estimate of $r_{F}$ is positive). All the stories in our data set had non-fan votes, giving all the estimates for $r_{N}$ as positive numbers. The line indicates where $r_{F}=r_{N}$.

the general user population, i.e., "niche interest" stories (corresponding to the upper left points in Fig. 18). As described below, this observation is useful to improve prediction of how popular a story will become based on reaction of early voters. Identifying niche interest stories could also aid user interface design by selectively highlighting stories on the friends interface that have particularly large estimated values of $r_{F}$. Stories with high ratios of $r_{F} / r_{N}$ tend to be promoted after fewer votes than those stories with low ratios.

An earlier study [24] noted a curious phenomenon: namely, stories that initially spread quickly through the network, i.e., receive a large proportion of early votes from fans, end up not becoming very popular; vice versa, stories that initially spread slowly through the fan network end up becoming popular. This phenomenon appears to be a generic feature of information diffusion on social networks and has also been observed on blog networks [9] and in Second Life [4].

Fig. 19 shows that our model explains this relationship, which arises from the difference in interestingness for fans and non-fans. Specifically, a low fraction of early votes by

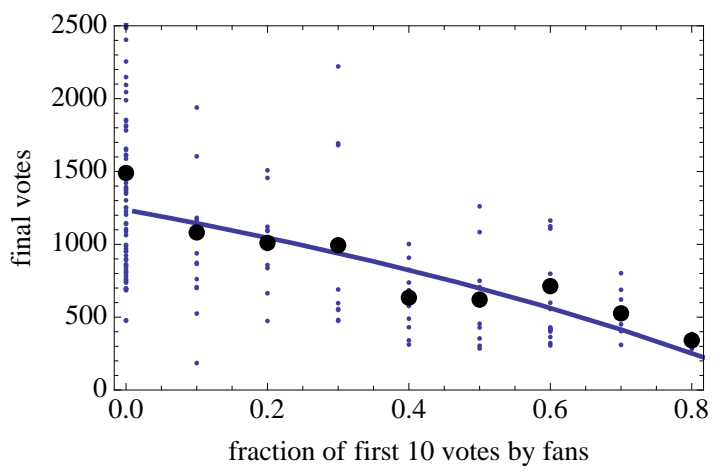

FIG. 19: Relation between final number of votes and the fraction of votes by fans among a story's first 10 votes. Small points are individual stories and the large points are the mean values for each number of votes by fans. The curve shows the model prediction.

fans indicates $r_{N}$ is relatively large to produce the early nonfan votes in spite of the lower visibility of upcoming stories to non-fan users. Once the story is promoted, it then receives relatively more votes from the general user community (most of whom are not fans of prior voters). The separation of effects of visibility and interestingness with our model improves this discrimination compared to just using the raw number of votes by fans and non-fans without regard for the story visibility at the time of the votes. For example, the correlation between the final number of votes and $r_{N} / r_{F}$ is 0.72 compared to 0.64 for the correlation between the final number of votes and $v_{N} / v_{F}$.

\section{Discussion}

This model with niche interests captures the consequences of link choices: people tend to become fans of users who submit or vote on stories of interest to that person. The ease of incorporating such additional detail is a useful feature of stochastic models.

Comparing the two models illustrates the practical challenges of incomplete or limited data. For example, data scraped from a web site can have errors due to unusual user names or unanticipated characters in story titles. Even when web sites provide an interface to collect data (as Digg provided after the data used in this paper was extracted), subtle differences in interpretation of the data fields can still arise, as when users who no longer have Digg account are all given the same name "inactive" and hence appear to be the same user if not specifically checked for in the script collecting the data.

In particular, the "law of surfing" parameter estimates for the two models are significantly different, a consequence of the log-likelihood being a fairly flat function of these parameters. This arises due to the relatively weak constraints that vote history provides on views, i.e., how many pages of upcoming or front page stories users choose to view during a visit to Digg. For stronger constraints on this behavior, data would ideally include the pages users actually viewed. While such data is in principle available via the web site access logs, this information is not publicly available for Digg. Similarly, the 
promotion algorithm used by Digg is deliberately not made public to reduce the potential for story submitters to game the system. To the extent that model parameter estimates differ from those that would be possible with this additional data, the stochastic approach identifies potential advantages models can provide the web site provider, with access to more precise data on user behavior, e.g., for predicting popularity of newly submitted stories. More generally, the sensitivity of parameters to the available measured data can suggest additional aspects of user behavior that would be most useful to determine, leading to more focus in future data collection and instrumentation of web communities. Alternatively, when the models indicate several different types of data could provide the required information, selecting the types most acceptable to the user community (e.g., privacy preserving) can facilitate the data collection while providing opportunities for more accurate models to guide the development of the web site and its usefulness to its community.

A related data quality issue is the length of time over which data is collected. On the one hand, collecting data for long periods can improve model parameter estimation by providing many more samples. On the other hand, web sites often rearrange or add features to their user interfaces, which change how users find content. Digg also occasionally changes the promotion algorithm. That is, the stochastic behavior associated with the site is nonstationary rather than arising from a fixed distribution. Moreover, over longer periods of time new users join the site and some users become inactive. Thus one can't simply improve the model parameter estimation by collecting data over longer periods of time [16, 37]. Instead, the models must be extended to include these additional timedependent behaviors.

In addition to improving quantitative estimation, similar qualitative behaviors seen with different models identify areas for further investigation. For example, in the two models presented here, the distribution of interestingness over the stories shows a lognormal distribution. This suggests there is an underlying multiplicative process giving rise to the observed values [31, 33]. Specifically, the lognormal distribution arises from the multiplication of random variables in the same way that the central limit theorem leads to the normal distribution from the addition of random variables under weak restrictions on their variance and correlations. Thus an important question raised by these models is identifying the story characteristics and user behaviors that combine multiplicatively to lead to the observed lognormal distributions. Identifying such properties would give a more detailed understanding of what leads to interesting content independent of the effects of visibility provided by the web site.

\section{MODEL-BASED PREDICTION}

As discussed above, predicting popularity in social media from intrinsic properties of newly submitted content is difficult [34]. However, users' early reactions provide some measure of predictability [16, 18, 24, 27, 35]. The early votes on a story allow us to estimate its interestingness to fans and

\begin{tabular}{cccc} 
& \multicolumn{2}{c}{ model } & direct \\
& distinct $r$ & same $r$ & extrapolation \\
\hline first 216 votes & $10 \%$ & $12 \%$ & $21 \%$ \\
first 10 votes & $18 \%$ & $23 \%$ & $29 \%$
\end{tabular}

TABLE IV: Prediction errors on whether a story receives at least 500 votes. The table compares three methods: 1) the full model which allows distinct values for $r_{F}$ and $r_{N}, 2$ ) the model constrained to have $r_{F}=r_{N}$, and 3) direct extrapolation from the rate the story accumulates votes. This comparison involves 178 promoted stories, of which 137 receive at least 500 votes.

other users. We can then use the model to predict how the story will accumulate additional votes. These predictions are for expected values and cannot account for the large variation due, for example, to a subsequent vote by a highly connected user which leads to a much larger number of votes.

As one prediction example, we evaluate whether a story will receive at least 500 votes. Predicting whether a story will attract a large number of votes, rather than the precise number of votes, is a useful criterion for predicting whether the story will "go viral" and become very popular. This is exactly Digg's intention behind using using crowd sourcing to select a subset of submitted content to feature on the front page [24]. The 500 vote threshold is a useful rule of thumb, as that is close to the median popularity value in a large sample of Digg stories [26, 38].

Table IV] compares the predictions with different methods, including a constrained version of our model with $r_{F}=r_{N}$, which assumes no systematic difference in interest between fans and other users.

We also compare with direct extrapolation from the early votes. In this procedure, with $v$ votes observed at time $t$, we extrapolate to $v t_{\text {final }} / t$, where we take $t_{\text {final }}$ to be 72 hours, a time by which stories have accumulated all, or nearly all, the votes they will ever get. We use a least squares linear fit between these observed and extrapolated values. A pairwise bootstrap test indicates the model has a lower prediction error than this extrapolation with $p$-value of $10^{-2}$.

This extrapolation method is similar to that used to predict final votes from the early votes [35], but with two differences: 1) we extrapolate from the time required for the story to acquire a given number of votes instead of the number of votes at a given time, and 2) we use early votes after submission (i.e., including when the story is upcoming, where the social network has a large effect) instead of early votes after promotion.

In the case of prediction based on the first 10 votes, which is before the stories are promoted, an additional question is how well the model predicts whether the story will eventually be promoted. We find a $25 \%$ error rate in predicting promotion based on the first 10 votes.

We can improve predictions from early votes by using the lognormal distributions of $r_{F}$ and $r_{N}$, shown in Fig. 16, as the prior probability to combine with the likelihood from the observations according to Bayes theorem. Specifically, instead of maximizing the likelihood of the observed votes, $P(r \mid$ votes $)$, as discussed above, this approaches 
a story with no fan votes

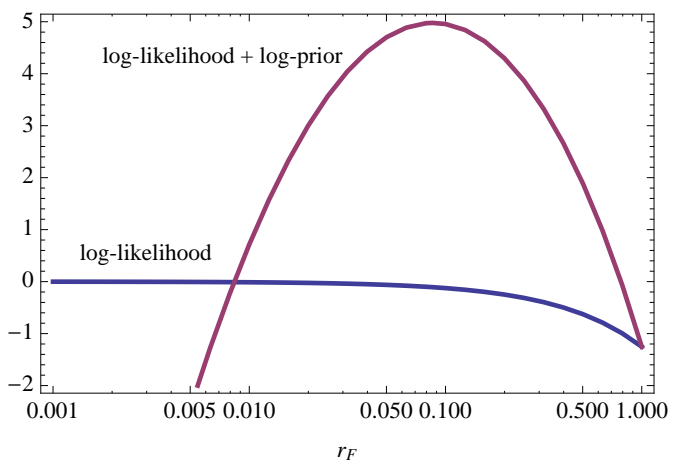

FIG. 20: Comparison of $\log$-likelihood (i.e., $\log P(r \mid$ votes $))$ and $\log$ likelihood plus $\log \left(P_{\text {prior }}(r)\right)$ for estimating $r_{F}$ for a story with no fan votes. The maximum of the log-likelihood is at $r_{F}=0$ while the maximum with the prior is $r_{F}=0.086$.

maximizes the posterior probability, which is proportional to $P(r \mid$ votes $) P_{\text {prior }}(r)$ where $P_{\text {prior }}$ is taken to be the lognormal distribution $P_{\text {lognormal }}$ in Eq. (9p) with parameters from the fits shown in Fig. 16 .

This method gives little change in estimates of $r_{N}$, due to the relatively large number of non-fan votes on each story. However, using the prior makes large changes in some of the $r_{F}$ estimates, thereby avoiding the small number of extreme predictions made by poor estimates. Using this prior to aid estimation is particularly significant when there are no votes by fans among the early votes, leading to an estimate of $r_{F}=$ 0 , but later a user with many fans votes on the story. In this case, as illustrated in Fig. 20, using the lognormal as a prior gives a positive estimate for $r_{F}$, thereby predicting some votes by any subsequent users who are fans of earlier voters.

By avoiding these extreme cases, this procedure improves the correlation between predicted and actual final votes as well as the predicted rank ordering of the stories (i.e., whether the story is likely to be relatively popular) as seen with a larger value of the Spearman rank correlation when using the prior distribution. For example, when predicting based on the first 10 votes, using this prior increases the Spearman rank correlation between predicted and actual number of votes from 0.46 to 0.53 . For comparison, this correlation for direct extrapolation from the first 10 votes is 0.32 and is 0.34 for the model constrained to have $r_{F}=r_{N}$. Pairwise bootstrap tests indicate the differences between these values are significant with $p$-values less than $10^{-3}$, except the difference between the last two cases has $p$-value of $10^{-2}$.

\section{RELATED WORK}

The Social Web provides massive quantities of available data about the behavior of large groups of people. Researchers are using this data to study a variety of topics, including detecting [1, 29] and influencing [11, 20] trends in public opinion, and dynamics of information flow in groups [28, 39].
Several researchers examined the role of social dynamics in explaining and predicting distribution of popularity of online content. Wilkinson [37] found broad distributions of popularity and user activity on many social media sites and showed that these distributions can arise from simple macroscopic dynamical rules. Wu \& Huberman [38] constructed a phenomenological model of the dynamics of collective attention on Digg. Their model is parameterized by a single variable that characterizes the rate of decay of interest in a news article. Rather than characterize evolution of votes received by a single story, they show the model describes the distribution of final votes received by promoted stories. Our model offers an alternative explanation for the distribution of votes. Rather than novelty decay, we argue that the distribution can also be explained by the combination of a non-uniform variations in the stories' inherent interest to users and effects of user interface, specifically decay in visibility as the story moves to subsequent front pages. Such a mechanism can also explain the distribution of popularity of photos on Flickr, which would be difficult to characterize by novelty decay. Crane \& Sornette [10] analyzed a large number of videos posted on YouTube and found that collective dynamics was linked to the inherent quality of videos. By looking at how the observed number of votes received by videos changed in time, they could separate high quality videos, whether they were selected by YouTube editors or spontaneously became popular, from junk videos. This study is similar in spirit to our own in exploiting the link between observed popularity and content quality. However, while this, and Wu \& Huberman study, aggregated data from tens of thousands of individuals, our method focuses instead on the microscopic dynamics, modeling how individual behavior contributes to the observed popularity of content. In [27] we used the simple model of social dynamics, reviewed in this paper, to predict whether Digg stories will become popular. The current paper improves on that work.

Researchers found statistically significant correlation between early and late popularity of content on Slashdot [18], Digg and YouTube [35]. Specifically, similar to our study, Szabo \& Huberman [35] predicted long-term popularity of stories on Digg. Through large-scale statistical study of stories promoted to the front page, they were able to predict stories' popularity after 30 days based on their popularity one hour after promotion. Unlike our work, their study did not specify a mechanism for evolution of popularity, and simply exploited the correlation between early and late story popularity to make the prediction. Our work also differs in that we predict popularity of stories shortly after submission, long before they are promoted. Several researchers [4, 9, 24] found that early diffusion of information across an interlinked community is a useful predictor of how far it will spread across the network in general. Both [24] and [9] exploited the anti-correlation between these phenomena to predict final popularity. Specifically, the former work used anti-correlation between the number of early fan votes and stories' eventual popularity on Digg to predict whether stories submitted by well connected users will become popular. That work exploited social influence only to make the prediction, and the results were not applicable to stories submitted by poorly connected users which were 
not quickly discovered by highly connected users. In contrast, the approach described in this paper considers effects of social influence regardless of the connectedness of the submitter, and also accounts for story quality in making a prediction about story popularity. An interesting open question is the nature of the social influence on voting. In our model, the influence has two components: increased visibility of a story to fans due to the friends interface and the higher interestingness of the story to fans. This higher interestingness could be due to self-selection, whereby users become fans of people whose submissions or votes are of particular interest. Alternatively, users could be directly influenced by the activities of others [34], with the possibility that this influence depends not just on whether friends vote on a story but also how many friends do so [7].

\section{CONCLUSION}

In the vast stream of new user-generated content, only a few items will prove to be popular, attracting a lion's share of attention, while the rest languish in obscurity. Predicting which items will become popular is exceedingly difficult, even for people with significant expertise. This prediction difficulty arises because popularity is weakly related to inherent content quality and social influence leads to an uneven distribu- tion of popularity that is sensitive to the early choices of users in the social network. We described how stochastic models of user behavior on a social media web site can partially address this prediction challenge by quantitatively characterizing evolution of popularity. The model shows how popularity is affected by item quality and social influence. We evaluated the usefulness of this approach for the social news aggregator Digg, which allows users to submit and vote on news stories. The number of votes a story accumulates on Digg shows its popularity. In earlier work we developed a model of social voting on Digg, which describes how the number of votes received by a story changes in time. In that model, knowing how interesting a story is to the user community, on average, and how connected the submitter is fully determines the evolution of the story's votes. This leads to an insight that a model can be used to predict story's popularity from the initial reaction of users to it. Specifically, we use observations of evolution of the number of votes received by a story shortly after submission to estimate how interesting it is, and then use the model to predict how many votes the story will get after a period of a few days. Model-based prediction outperforms other methods that exploit social influence only, or correlation between early and late votes received by stories. We improved prediction by developing a more fine-grained model that differentiates between how interesting a story is to fans and to the general population.
[1] E. Adar, L. Zhang, L. A. Adamic, and R. M. Lukose. Implicit structure and the dynamics of blogspace. In Workshop on the Weblogging Ecosystem, 13th International World Wide Web Conference, 2004.

[2] Nitin Agarwal, Huan Liu, Lei Tang, and Philip S. Yu. Identifying the influential bloggers in a community. In WSDM '08: Proceedings of the international conference on Web search and web data mining, pages 207-218, New York, NY, USA, 2008. ACM.

[3] Chris Anderson. The Long Tail: Why the Future of Business is Selling Less of More. Hyperion, 2006.

[4] E. Bakshy, B. Karrer, and L. A. Adamic. Social influence and the diffusion of user-created content. In EC '09: Proc. 10th ACM conference on Electronic commerce, pages 325-334, 2009.

[5] Albert-Laszlo Barabási. The origin of bursts and heavy tails in human dynamics. Nature, 435:207-211, May 2005.

[6] M. G. Bulmer. On fitting the Poisson lognormal distribution to species-abundance data. Biometrics, 30:101-110, 1974.

[7] Damon Centola. The spread of behavior in an online social network experiment. Science, 329:1194-1197, 2010.

[8] Aaron Clauset, Cosma Rohilla Shalizi, and M. E. J. Newman. Power-law distributions in empirical data. SIAM Review, 51:661-703, 2009.

[9] Richard Colbaugh and Kristin Glass. Early warning analysis for social diffusion events. In Proceedings of IEEE International Conferences on Intelligence and Security Informatics, 2010.

[10] R. Crane and D. Sornette. Viral, quality, and junk videos on youtube: Separating content from noise in an information-rich environment. In Proc. of AAAI symposium on Social Information Processing, Menlo Park, CA, 2008. AAAI.
[11] P. Domingos and M. Richardson. Mining the network value of customers. In Proc. of KDD, 2001.

[12] Bradley Efron. Bootstrap methods: Another look at the jackknife. Annals of Statistics, 7:1-26, 1979.

[13] Vicente Gómez, Andreas Kaltenbrunner, and Vicente López. Statistical analysis of the social network and discussion threads in Slashdot. In $W W W$ '08: Proceeding of the 17th international conference on World Wide Web, pages 645-654, New York, NY, USA, 2008. ACM.

[14] Joseph M. Hilbe. Negative Binomial Regression. Cambridge Univ. Press, 2008.

[15] Tad Hogg and Kristina Lerman. Stochastic models of usercontributory web sites. In Proceedings of the Third International Conference on Weblogs and Social Media (ICWSM2009), pages 50-57, 2009.

[16] Tad Hogg and Gabor Szabo. Diversity of user activity and content quality in online communities. In Proc. of the Third International Conference on Weblogs and Social Media (ICWSM2009), pages 58-65. AAAI, 2009.

[17] Bernardo A. Huberman, Peter L. T. Pirolli, James E. Pitkow, and Rajan M. Lukose. Strong regularities in World Wide Web surfing. Science, 280:95-97, 1998.

[18] A. Kaltenbrunner, V. Gomez, and V. Lopez. Description and prediction of slashdot activity. In Proc. 5th Latin American Web Congress (LA-WEB 2007), 2007.

[19] N. G. Van Kampen. Stochastic Processes in Physics and Chemistry. Elsevier Science, Amsterdam, revised and enlarged edition, 1992.

[20] David Kempe, Jon Kleinberg, and Éva Tardos. Maximizing the spread of influence through a social network. In $K D D$ '03: Proceedings of the ninth ACM SIGKDD international confer- 
ence on Knowledge discovery and data mining, pages 137-146, New York, NY, USA, 2003. ACM.

[21] A. Kittur, E. Chi, Bryan A. Pendleton, Bongwon Suh, and T. Mytkowicz. Power of the few vs. wisdom of the crowd: Wikipedia and the rise of the bourgeoisie. In Proceedings of World Wide Web Conference, 2006.

[22] K. Lerman. Social information processing in social news aggregation. IEEE Internet Computing: special issue on Social Search, 11(6):16-28, 2007.

[23] K. Lerman. Social networks and social information filtering on digg. In Proc. of International Conference on Weblogs and Social Media (ICWSM-07), 2007.

[24] K. Lerman and A. Galstyan. Analysis of social voting patterns on digg. In Proceedings of the 1st ACM SIGCOMM Workshop on Online Social Networks, 2008.

[25] K. Lerman and L. Jones. Social browsing on flickr. In Proc. of International Conference on Weblogs and Social Media (ICWSM-07), 2007.

[26] Kristina Lerman and Rumi Ghosh. Information contagion: an empirical study of spread of news on digg and twitter social networks. In Proceedings of 4th International Conference on Weblogs and Social Media (ICWSM), May 2010.

[27] Kristina Lerman and Tad Hogg. Using a model of social dynamics to predict popularity of news. In Proceedings of 19th International World Wide Web Conference (WWW), 2010.

[28] J. Leskovec, L. Adamic, and B. Huberman. The dynamics of viral marketing. ACM Transactions on the Web, 1(1), 2007.

[29] Jure Leskovec, Andreas Krause, Carlos Guestrin, Christos Faloutsos, Jeanne Vanbriesen, and Natalie Glance. Costeffective outbreak detection in networks. In KDD '07: Proceedings of the 13th ACM SIGKDD international conference on Knowledge discovery and data mining, pages 420-429, New
York, NY, USA, 2007. ACM.

[30] Guthrie Miller. Statistical modelling of Poisson/log-normal data. Radiation Protection Dosimetry, 124:155-163, 2007.

[31] Michael Mitzenmacher. A brief history of generative models for power law and lognormal distributions. Internet Mathematics, 1:226-251, 2004.

[32] Manfred Opper and David Saad, editors. Advanced Mean Field Methods: Theory and Practice. MIT Press, Cambridge, MA, 2001.

[33] S. Redner. Random multiplicative processes: An elementary tutorial. American Journal of Physics, 58:267-273, 1990.

[34] M.J. Salganik, P.S. Dodds, and D.J. Watts. Experimental study of inequality and unpredictability in an artificial cultural market. Science, 311:854, 2006.

[35] Gabor Szabo and Bernardo A. Huberman. Predicting the popularity of online content. Communications of the ACM, 53(8):80-88, 2010.

[36] A. Vázquez, J. G. Oliveira, Z. Dezsö, K.-I. Goh, I. Kondor, and A.-L. Barabási. Modeling bursts and heavy tails in human dynamics. Phys. Rev. E, 73(3):036127+, March 2006.

[37] Dennis M. Wilkinson. Strong regularities in online peer production. In EC '08: Proceedings of the 9th ACM conference on Electronic commerce, pages 302-309, New York, NY, USA, 2008. ACM.

[38] Fang Wu and Bernardo A. Huberman. Novelty and collective attention. Proceedings of the National Academy of Sciences, 104(45):17599-17601, November 2007.

[39] Fang Wu, Bernardo A. Huberman, Lada A. Adamic, and Joshua R. Tyler. Information flow in social groups. Physica A: Statistical and Theoretical Physics, 337(1-2):327-335, June 2004.

[40] $\Theta(x)$ is a step function: 1 when $x \geq 0$ and 0 when $x<0$. 